\title{
Instantaneous wavenumber estimation for damage quantification in layered plate structures
}

\author{
Olivier Mesnil ${ }^{a}$, Cara A.C. Leckey ${ }^{b}$, and Massimo Ruzzene ${ }^{c}$ \\ ${ }^{a}$ D. Guggenheim School of Aerospace Engineering, Georgia Institute of Technology, Atlanta, \\ GA, 30332, US \\ ${ }^{b}$ NASA Langley Research Center, Hampton, VA, 23681, US \\ ${ }^{c}$ D. Guggenheim School of Aerospace Engineering and G. W. Woodruff School of Mechanical \\ Engineering, Georgia Institute of Technology, Atlanta, GA, 30332, US
}

\begin{abstract}
Guided wavefield detection is at the basis of a number of promising techniques for the identification and the characterization of damage in plate structures. Among the processing techniques proposed, the estimation of instantaneous and local wavenumbers can lead to effective metrics that quantify the extent of delaminations in composite plates. This paper illustrates the application of instantaneous and local wavenumber damage quantification techniques for high frequency guided wave interrogation. The proposed methodologies can be considered as first steps towards a hybrid structural health monitoring/nondestructive evaluation approach for damage assessment in composites.
\end{abstract}

Keywords: Structural health monitoring, Damage detection in composites plates, Wave propagation

\section{INTRODUCTION}

Guided wavefield detection is at the basis of a number of promising techniques for the identification and the characterization of damage in plate structures. With the continued and expanded use of composite materials in aerospace applications, techniques that can provide quantitative and detailed damage information are needed. Guided wave methods have long been investigated as a promising approach for structural health monitoring (SHM) applications in plate-like structures, and progress has been made in using guided waves to locate damage in composites. ${ }^{1,2}$ In SHM techniques, a small number of in-situ sensors are used to excite and receive guided waves. A proposed concept of hybrid SHM/nondestructive evaluation (NDE) is combining guided wave excitation via in-situ SHM sensors with scanning laser Doppler vibrometry to yield the detailed damage information that is required for prognostics (e.g., feeding detailed damage information into damage progression models for remaining life predictions). Advanced processing techniques can be applied to wavefield data in order to reveal damage information. Among the guided wavefield processing techniques proposed, the estimation of instantaneous and local wavenumbers can lead to effective metrics that quantify the extent of delamination damage in composite plates.

This paper illustrates the application of instantaneous and local wavenumber damage quantification techniques for high frequency guided wave interrogation. The two techniques are first outlined in section 2 and then applied to simulated and experimental wavefield data, as described in sections 3 and 4 . The proposed methodologies can be considered as first steps toward a hybrid structural SHM/NDE approach for damage assessment in composites.

Further author information: (Send correspondence to Olivier Mesnil) E-mail: omesnil3@gatech.edu 


\section{THE INSTANTANEOUS AND LOCAL WAVENUMBER}

\subsection{The Frequency Domain Instantaneous Wavenumber}

\subsubsection{The Instantaneous Wavenumber}

For a recorded wavefield, the Instantaneous Wavenumber (IW) is a local function of the wavenumber at any instant of time. For a 1D problem, the IW is defined as the spatial derivative of the phase of the complex wavefield. In order to avoid phase unwrapping, the IW is computed by Equation 1 where $g(x, t)$ is the Hilbert transform of the displacement function of the wavefield and $k$ is its wavenumber. The mathematical process is fully described by Rogge and Parker. ${ }^{3}$ Since the IW is computed using a derivative, the effects of the noise are amplified, therefore a median filter is applied to every IW map.

$$
k(x, t)=i m a g\left(\frac{\frac{\partial g(x, t)}{\partial x}}{g(x, t)}\right)
$$

For a $2 \mathrm{D}$ case, the IW is given by $I W(x, y, t)=\sqrt{k_{x}^{2}(x, y, t)+k_{y}^{2}(x, y, t)}$.

In a general case, the IW is a function of the wavenumbers of all the excited modes. However, if only one mode is present at a given spatial point and time, the IW is a direct measure of the wavenumber of that mode. Moreover, if several modes are superimposed but one of them is dominant (higher amplitude and/or wavenumber), the IW is a measure of the wavenumber of the dominant mode only. This is, in general, the case when only the A0 (asymmetric) and the S0 (symmetric) modes are created due to an excitation, since the amplitude and the wavenumber of the A0 mode are higher than the S0 mode.

Figure 1b is the IW mapping for artificial data (Figure 1a) created using wavepackets of 5 and $11 \mathrm{rad} / \mathrm{m}$. The IW directly measures the wavenumbers of the wavepackets since they are spatially separated.

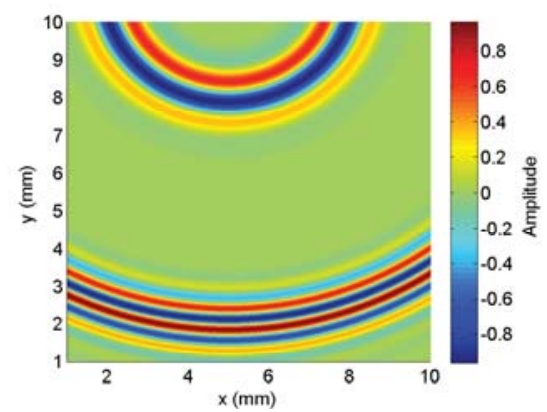

(a)

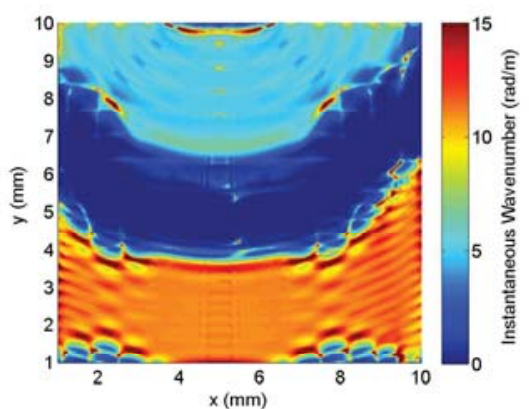

(b)

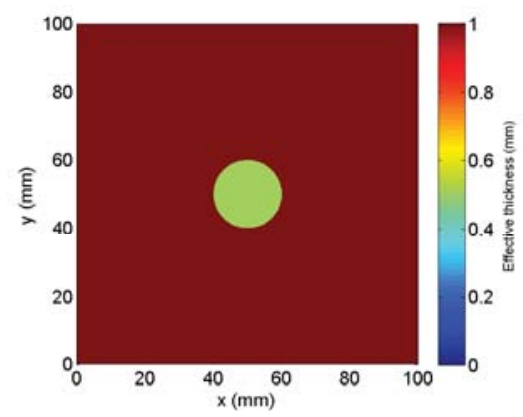

(c)

Figure 1: (a): Artificial data with wavepackets of 5 and $11 \mathrm{rad} / \mathrm{m}$, (b): IW map associated to the artificial data and (c): Theoretical Effective thickness of plate with a circular delamination

\subsubsection{Effective Thickness}

The Effective Thickness defines the subsurface depth of any features such as delaminations. For a pristine plate, the Effective Thickness is a constant equal to the thickness of the plate. For a plate with a local subsurface feature, the Effective Thickness is a local value equal to the depth from the measurement surface to the feature at its location and equal to the thickness of the plate elsewhere. The theoretical Effective Thickness mapping of a plate with a mid-thickness circular delamination at its center is shown in Figure 1c.

The wavenumber is related to the Effective Thickness using dispersion relations (Figure 2a). For a layered plate, as the features are more likely to be found between the lamina, the Effective Thickness is a discrete quantity with $N-1$ possible values, where $\mathrm{N}$ is the number of lamina. 
The variations of the wavenumber of the A0 and the S0 modes for an 8 ply composite glass fiber plate as a function of the Effective Thickness at $100 \mathrm{kHz}$ are plotted in Figure 2b. It is important to note that the wavenumber of the A0 mode would increase over the delaminated area while the wavenumber of the S0 mode would decrease. However, since the A0 mode is dominant compared to the S0 mode, a local increase in the IW mapping is expected in the featured area.

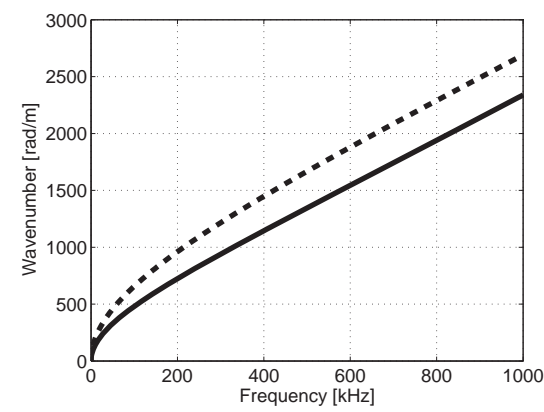

(a)

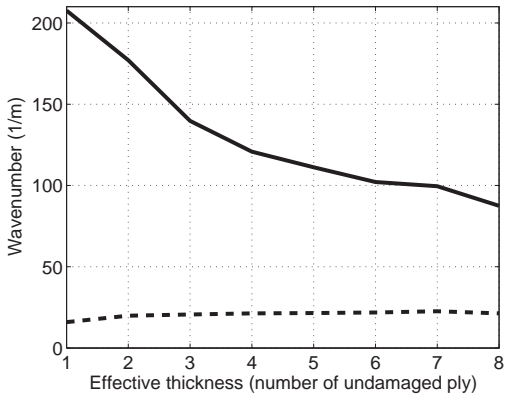

(b)

Figure 2: (a): Dispersion relation of the A0 mode for a $1 \mathrm{~mm}$ thick (Dashed line) and $2 \mathrm{~mm}$ thick (Solid line) aluminium plate and (b): Aluminium dispersion relations and Wavenumber vs Effective Thickness for S0

(dashed line) and A0 (solid line) modes of an $1.6 \mathrm{~mm}$ thick glass fiber composite plate

\subsubsection{The Frequency Domain Instantaneous Wavenumber}

The IW is a very powerful tool used to compute a wavenumber map from a wavefield at a given instant of time. Since only a frequency and a wavenumber are needed to determine the local Effective Thickness, knowing the wavenumber at each instant of time is unnecessary. Therefore, the IW algorithm is used in the frequency domain. To calculate the IW in the frequency domain, a time Fourier transform is applied to the wavefield, then the dominant frequency is extracted by selecting the frequency with the highest displacement amplitudes (dominant response frequency). Eventually the IW is applied in this frequency-space domain. To illustrate this concept, a numerical simulation of a $200 \mathrm{~mm} \times 200 \mathrm{~mm} \times 1.6 \mathrm{~mm}$ glass fiber composite plate of layup $[0 / 90 / \pm 45]_{s}$ containing a $\varnothing 25 \mathrm{~mm}$ delamination between the second and third ply is implemented with a 100 $\mathrm{kHz} 4$ cycles sine burst excitation. The out-of-plane displacement is recorded during a sufficiently long time to observe the first reflection on the edges of the plate. As both the S0 and A0 modes are excited, those modes are superimposed in the Frequency-Space domain. However, as the A0 mode is the dominant mode, the IW is a measure of the A0 wavenumber only. Therefore, only the Wavenumber vs Effective Thickness curve for the A0 mode is used to convert the IW map into the Effective Thickness map. Figure 3a depicts the frequency-space domain representation of this wavefield at the dominant propagation frequency $(73 \mathrm{kHz})$ and Figure 3b is the IW mapping associated with the previous picture. The irregular pattern in the frequency-space domain representation immediately highlights the circular feature and the significant increase in the IW mapping accurately provides the in plane coordinate of the subsurface feature. The whole IW map is converted into an Effective Thickness mapping (see Figure 3c) by looking for the best match between each individual value of the IW mapping and the dispersion relations. The estimated Effective Thickness map (Figure 3c) is very similar to the simulated values (Figure 3d), validating the process in this simple low-noise case.

\subsection{The Local Wavenumber}

The local wavenumber (LW) technique can be applied to time domain wavefield data to yield a spatial map of the localized wavenumber (length versus width versus wavenumber). The LW approach requires wavefield data at all points in time within a chosen time-gating (which may, for example, be the time capturing a certain mode interacting with damage). The LW method has previously been successfully applied to experimental wavefield data to characterize near-surface impact damage in CFRP composite specimens. ${ }^{4}$ The localized spatial aspect of the technique not only allows for a non-contact measure of the damage size; but also allows for 


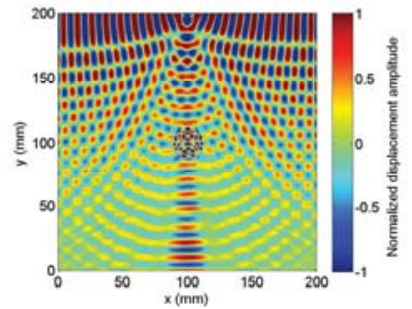

(a)

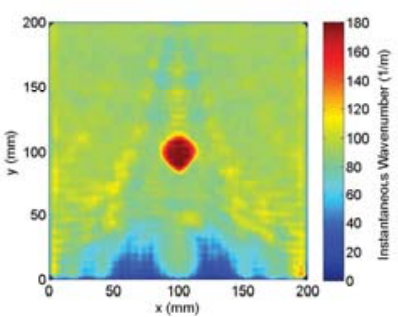

(b)

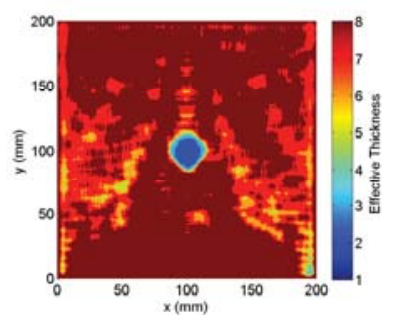

(c)

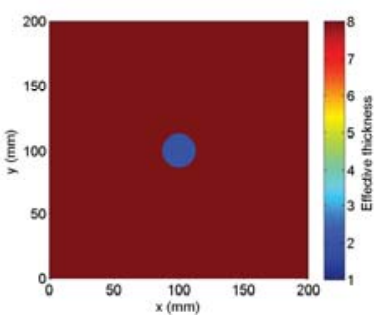

(d)

Figure 3: (a): Frequency-Space domain, (b): IW map, (c): Effective Thickness map and (d): Theoretical Effective Thickness map

a measure of delamination depth for near-surface "non-shadowed" damage (i.e., for delaminations occurring beneath one another, only the uppermost delamination depth can be resolved - a characteristic of both IW and LW techniques). As described in section 2.1.2, since wavenumbers are directly related to thickness, the LW technique can be used to determine the depth of delamination damage (i.e., the thickness above delamination damage). The method requires applying a 3D Fourier transform (FFT) to spatially windowed regions of the time domain wavefield data (a process analogous to a short-time Fourier transform) to yield space-wavenumber information.

The LW technique is implemented by first using a 2D Hann window to select a local 2D spatial region of the wavefield for all points in time. Next a 3D FFT is applied to the windowed space-time data to yield wavenumber-frequency data for that specific spatial region. The frequency slice corresponding to the center excitation frequency is then selected and the dominant wavenumber, at that frequency and spatial window location, is calculated using a weighted mean. The resulting wavenumber is plotted as the pixel value at the position corresponding to the center of the window location, as shown in the diagram in figure 4 . The next window position is then chosen and the process repeated to build up a $2 \mathrm{D}$ space-wavenumber plot. Note that neighboring spatial window positions can be chosen such that windows overlap. A lengthier description of the mathematics involved in the local wavenumber method can be found in prior work by Rogge and Leckey. ${ }^{4}$

The need to choose a spatial window size is a key difference between the IW approach and the LW technique. The spatial window size should be at least twice the guided wave mode wavelength to accurately resolve the local wavenumber. The window size is also directly correlated to the spatial resolution of the resulting wavenumber map (larger window sizes result in worse spatial resolution). Shorter wavelength guided wave modes will therefore result in a higher resolution damage map since a smaller window size can be used while still resolving the full wavelength. Since the damage depth is not known a-priori in a real setting, the most ideal window size (twice the wavelength of the chosen guided wave mode as it passes over the damage) is also not known ahead of time. A sweep in window size can be implemented in this situation, as described by Rogge and Leckey. ${ }^{4}$ In section 3 , we have chosen to adopt a window size that is slightly less than twice the wavelength of the A0 mode in the full plate thickness, since that value is known a-priori and results in a window size that would not be too small to the capture wavenumber of the decreased wavelength A0 mode that would exist above delamination damage for that case. Section 4 shows results for two different window sizes.

\section{NUMERICAL RESULTS}

\subsection{Simulation}

The 3D elastodynamic finite integration technique (EFIT) was implemented to model wave interaction with realistic, complex geometry delamination damage. The custom modeling code is designed to run efficiently on multicore and cluster computing systems. Previous work by Leckey et. al. presents details of the anisotropic EFIT approach as well as validation comparisons of the 3D anisotropic EFIT code results to experiment and theory. ${ }^{5}$ The guided wave simulations implemented for this paper incorporate ply-level material properties and a realistic 3D volumetric delamination, as shown in figure 5a. The delamination damage was directly read into the simulation from a 3D damage map created using 23.4 micron resolution microfocus x-ray computed tomography 


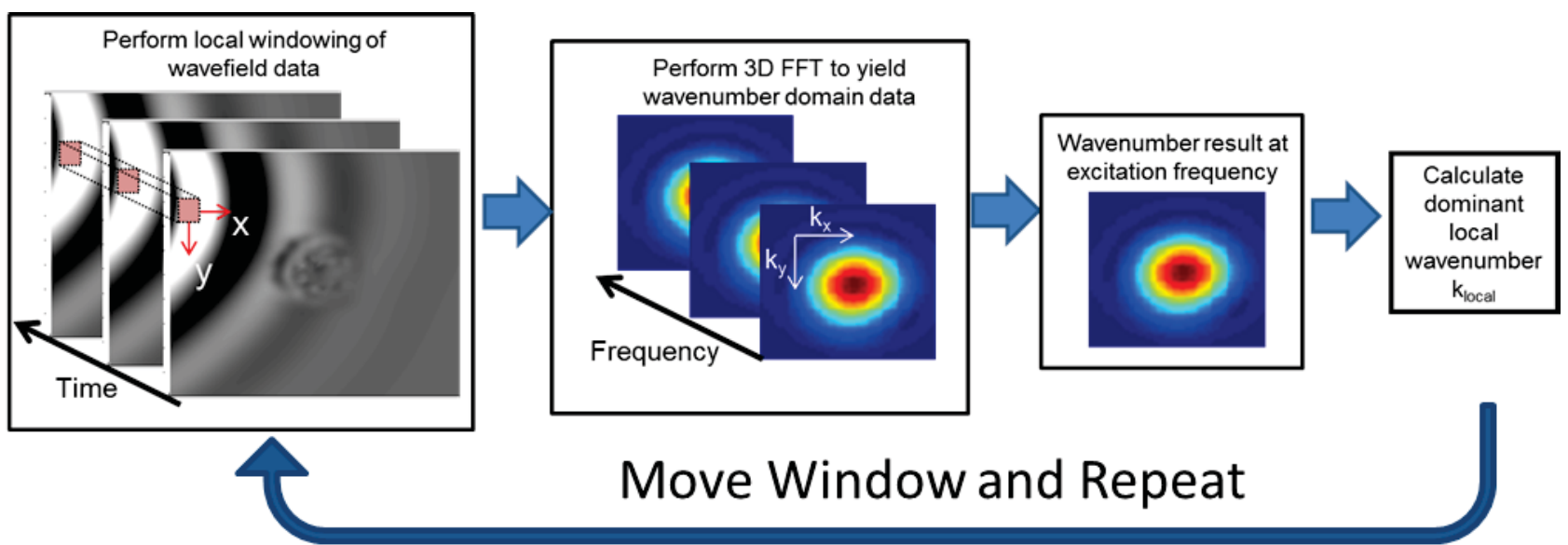

Figure 4: Diagram showing the local wavenumber process.

(X-ray CT) data from a composite laminate sample containing impact damage. The spatial step size used in the simulation was set equal to the X-ray $\mathrm{CT}$ data resolution in order to capture details of the delamination geometry.

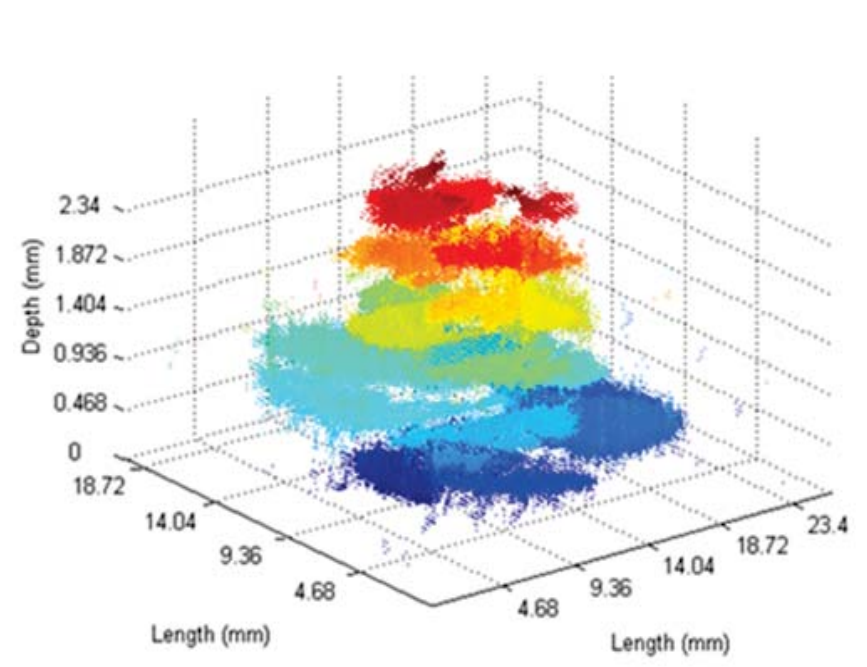

(a)

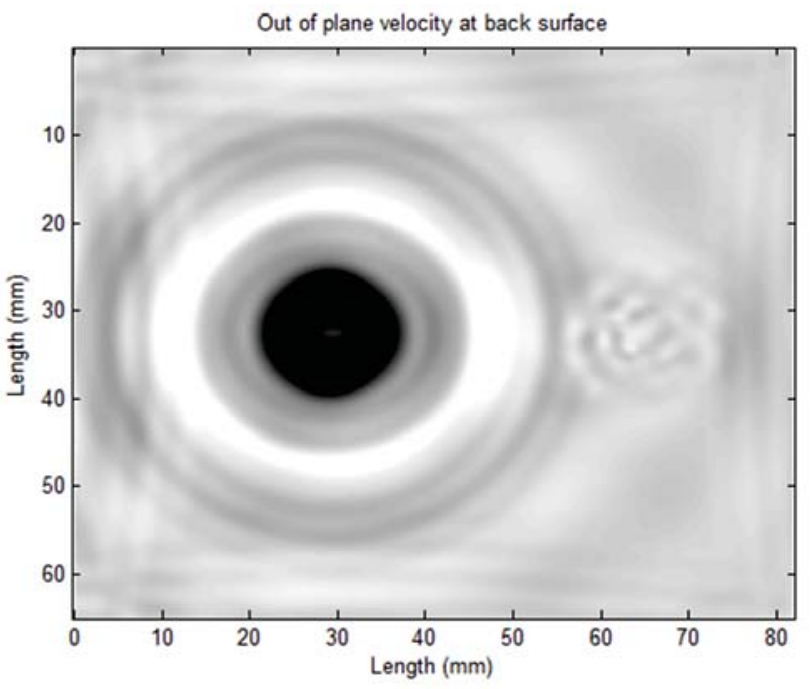

(b)

Figure 5: (a): Volumetric delamination damage from x-ray CT scans of a composite sample containing impact damage and (b): A snapshot in time of the fastest guided wave mode (S0) interacting with the delamination damage at the composite surface (out-of-plane velocity).

The 3D EFIT simulations calculate the out-of-plane and in-plane wavefield throughout the composite sample. Out-of-plane velocity at the composite sample surface can be output from the simulation and is analogous to the velocity wavefield data that can be collected experimentally using a scanning one-dimensional (1D) laser Doppler vibrometer. In this paper we focus on out-of-plane wave behavior, but in general, realistic guided wave simulations create the capability for cost-effective studies of in-plane and out-of-plane wave behavior throughout the composite.

The properties of the simulated composite match those of the real impact damaged sample. The composite laminate is made up of IM7/8552 prepreg material with a 26 ply thick layup of $[0 /+45 /-45 / 90]_{s}$. Details of the material properties are available in prior papers by Leckey. ${ }^{5}$ The full simulated sample size is $82 \mathrm{~mm}$ by 65 
mm by $3.23 \mathrm{~mm}$. A $400 \mathrm{kHz} 5$-cycle Hann windowed sine wave was excited over a 0.5 inch circular region on the simulated composite sample. At this frequency-thickness several guided wave modes exist. A snapshot in time of the fastest guided wave mode ( $\mathrm{S} 0$ ) interacting with the delamination damage is shown in figure 5b. Note that mode conversion can be observed from S0 to A0 at the plate edges. In the following sections we discuss the application of the IW and LW techniques to the simulated wavefield data.

\subsection{Application of the Frequency Domain Instantaneous Wavenumber}

Using the IW technique described in Section 2.1 on the out-of-plane simulation data, the IW map is computed at the dominant frequency $422 \mathrm{kHz}$ and plotted in figure 6c. The IW map can be compared with the actual geometry in figure 7 (right). The estimated shape and size of the delamination are very similar to the X-ray CT data. However, damage region is larger (more spread out) and the edges of the damage are less accurately located than in the result of the LW technique (see following section). Furthermore, the $400 \mathrm{kHz}$ excitation frequency generates not only A0 and S0 modes in the simulated composite, but A1 and S1 modes are also excited in the pristine plate region (see figure 6a and 6b). In this case, the A0 mode is not dominant and an Effective Thickness map is not as easily deduced from the IW map.

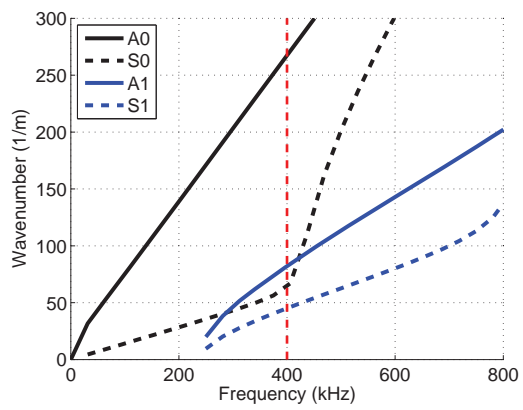

(a)

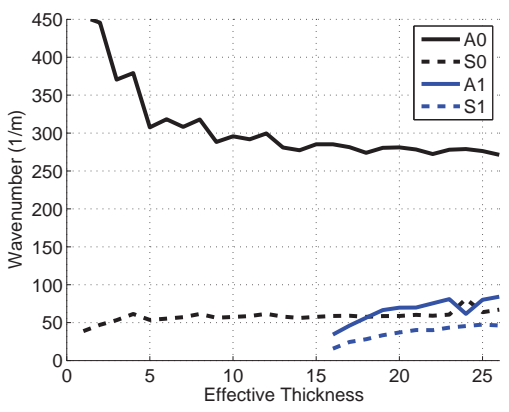

(b)

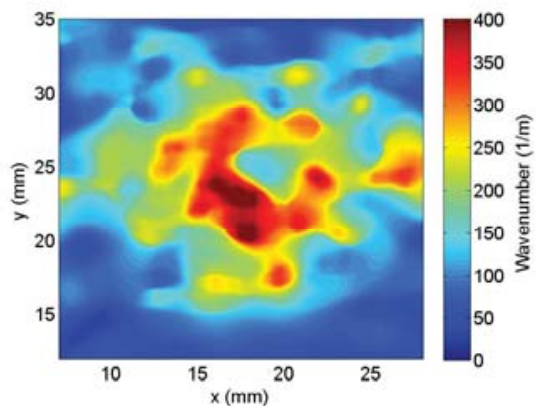

(c)

Figure 6: (a): Dispersion relation for a pristine plate: Wavenumber vs Frequency, (b): Dispersion relation at $400 \mathrm{kHz}$ : Wavenumber vs Effective thickness and (c): IW map

\subsection{Application of the Local Wavenumber}

The LW technique was applied to the simulated out-of-plane wavefield data using a window size of $5.6 \mathrm{~mm}$. Since the A0 mode has a smaller wavelength than the faster S0 mode, and thus will provide better spatial resolution for the wavenumber map, the LW technique was applied to wavefield data for a time gate encompassing the A0 mode interaction with the delamination damage. The results of applying the LW technique are shown in figure 7 next to an image showing the damage details from X-ray CT data. Note that figure 7 shows the LW result for a spatial region surrounding the delamination. As shown in the figure, the damage area from the LW result matches fairly well with the X-ray CT data, with a size of approximately $18.35 \mathrm{~mm}$ by $16.10 \mathrm{~mm}$ shown in the LW result versus approximately $18.72 \mathrm{~mm}$ by $14.82 \mathrm{~mm}$ in the CT data. The multi-mode situation is more complicated than the simplified case in figure 3 . The dispersion curves can still be used to relate the wavenumber values to delamination depth, and is planned for future work.

\section{EXPERIMENTAL RESULTS}

\subsection{The Experimental Setup}

Experiments have been conducted in order to further study the IW and LW techniques and to confirm the techniques using a simplified damage geometry, compared to the complex damage geometry of the numerical simulation in the previous section. The experimental setup uses a $600 \mathrm{~mm}$ x $600 \mathrm{~mm} \times 1.6 \mathrm{~mm}$ glass fiber S2 composite plate of layup $[0 / 90 / \pm 45]_{s}$ with a $\varnothing 25 \mathrm{~mm}$ delamination (teflon insert) between the $2^{\text {nd }}$ and the $3^{\text {rd }}$ ply manufactured at the Dipartimento di Ingegneria Aerospaziale of the Politecnico di Milano, Italy. The plate 

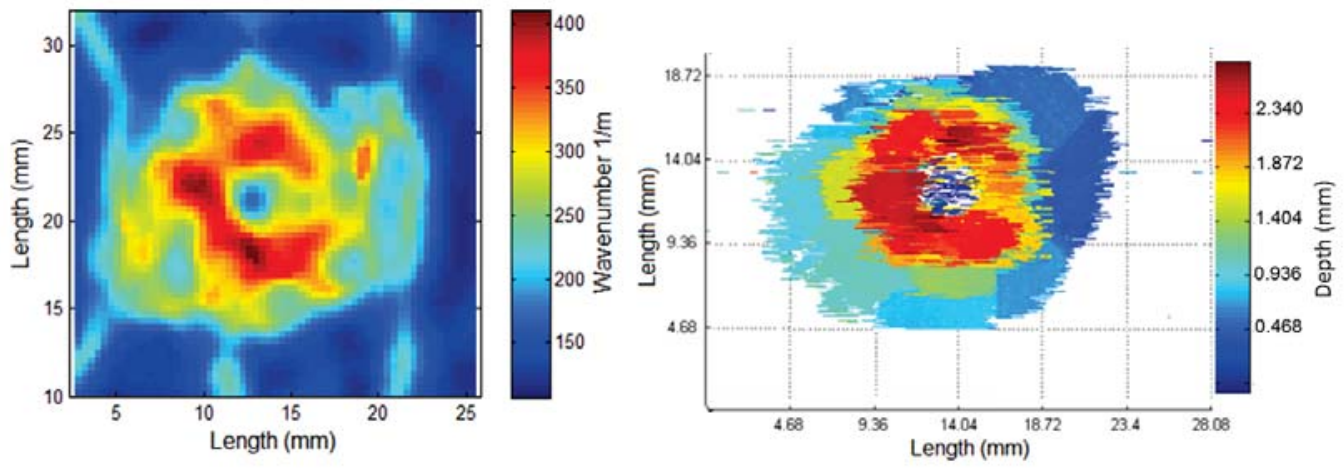

Figure 7: Left: Local wavenumber result for a spatial region surrounding the delamination, using wavefield data from the composite surface where the impact occurred. Right: X-ray CT data showing the delamination damage viewed from the impacted side of the sample.

is excited by a piezo transducer located at $100 \mathrm{~mm}$ from the delaminated area. The out of plane velocity is recorded by a scanning vibrometer Polytec PSV-400 using the Doppler effect. The experimental setup is similar to the one used in reference. ${ }^{6}$ The excitation is a $100 \mathrm{kHz} 4$ cycles sine burst. The scanned area is centered on the delamination region. At this excitation frequency, only the A0 and S0 modes are excited and since their velocities are different, the modes do not overlap in the scanned area. The wavefield is recorded until the A0 mode reaches the edges of the plate. Figures 8 show the interaction of the S0 and the A0 modes with the delaminated area. The amplitude of the A0 mode is about 100 times greater than the one of the S0 mode and its wavenumber is about 5 times larger, therefore the Frequency-Space representation is dominated by the A0 mode and the IW is a measure of the Wavenumber of the A0 mode.

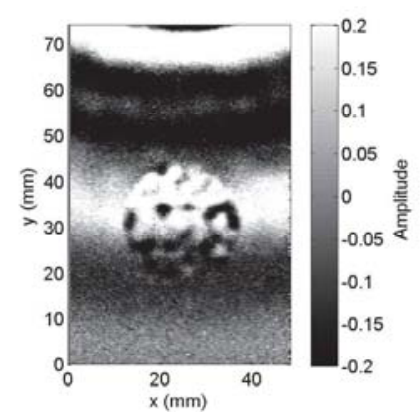

(a) S0 mode

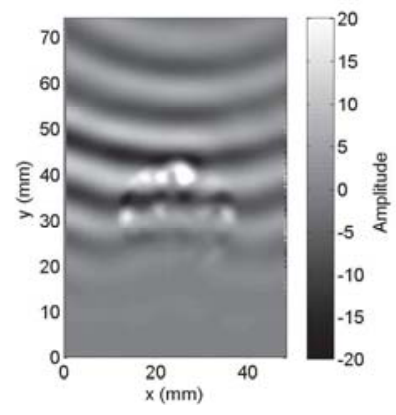

(b) A0 mode

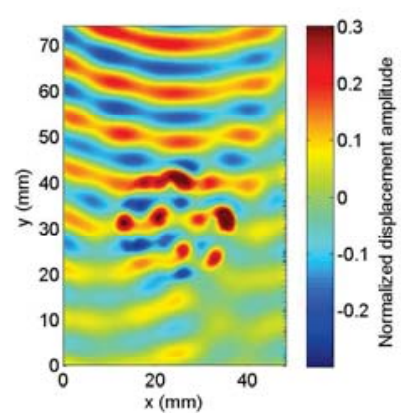

(c)

Figure 8: (a) and (b): Interaction of the $\mathrm{S} 0$ and $\mathrm{A} 0$ modes with the delamination and (c): Frequency-Space domain

\subsection{Application of the Frequency Domain Instantaneous Wavenumber}

The dominant frequency of the experimentally recorded wavefield is $93 \mathrm{kHz}$. The frequency-space representation of the wavefield is shown in Figure 8c and its associated IW mapping in Figure 9a

As the feature is hardly distinguishable in the frequency-space representation, the IW mapping clearly highlights the shape of the delaminated area. Using the dispersion relations of the studied plate, the Effective Thickness map (Figure 9b) is created from the IW map. The theoretical Effective Thickness map is represented in Figure 9c. In this case, the frequency domain instantaneous wavenumber provides a correct estimation of the in plane size of the feature but the out-of-plane coordinate (depth) is slightly overestimated since the estimated depth of the feature is approximately 3 ply and the actual depth is 2 ply. 


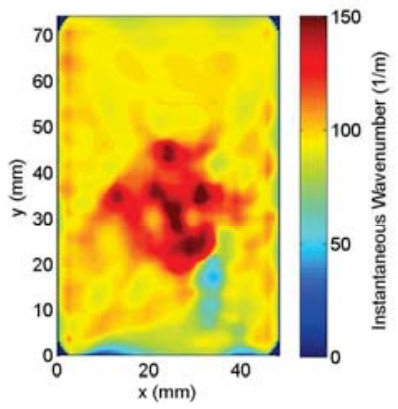

(a)

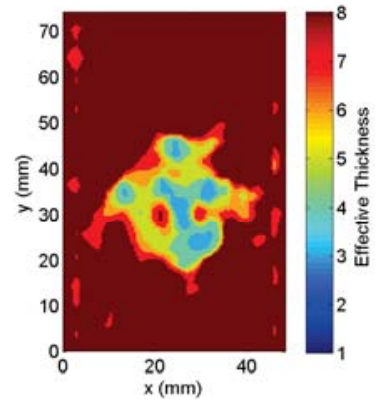

(b)

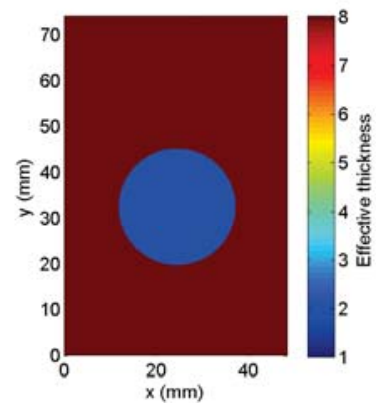

(c)

Figure 9: (a): IW map, (b): Effective Thickness map and (c): Theoretical Effective Thickness map

\subsection{Application of the Local Wavenumber}

The LW technique was also applied to the experimental wavefield data. The results show that, as expected, the LW technique is not ideal for cases where the wavelength of generated guided wave modes is on the order of, or larger than, the damage size. As mentioned in section 2.2, the local wavenumber technique gives an accurate wavenumber result when the spatial window size is equal to or greater than twice the wavelength of the propagating guided wave mode. Additionally, the smaller the guided mode wavelength, the better the spatial resolution of the resulting local wavenumber plot and of any damage that may be present.

Figures 10a and 10b show the resulting local wavenumber plots for two different spatial window sizes: 1) a $5 \mathrm{~mm}$ window size, which is smaller than the guided mode wavelengths but more ideal for resolving spatial wavenumber changes, and 2) a $20 \mathrm{~mm}$ window size, which is around twice the wavelength of the A0 guided wave mode in the full plate and thus better at resolving wavenumber while worse at resolving spatial changes. As can be seen in the figures, the smaller window more accurately captures the damage location and size, while the larger window size more accurately captures the delamination depth (comparing figure 10b to figure 9a). Note that the dark blue oval region on the right side of the $20 \mathrm{~mm}$ result is likely due to noise in the original experimental data, which affects the wavenumber calculation that occurs during the LW process.

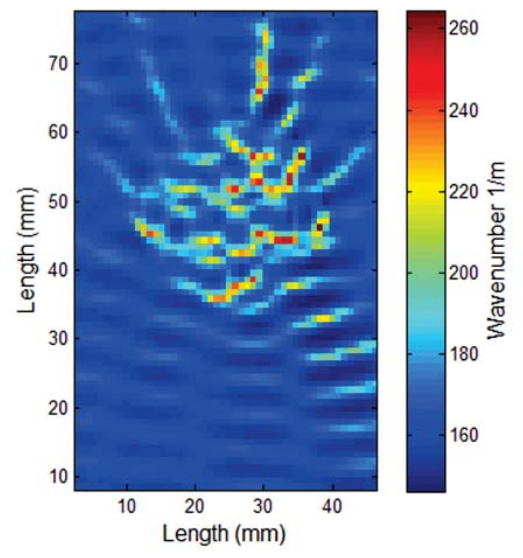

(a)

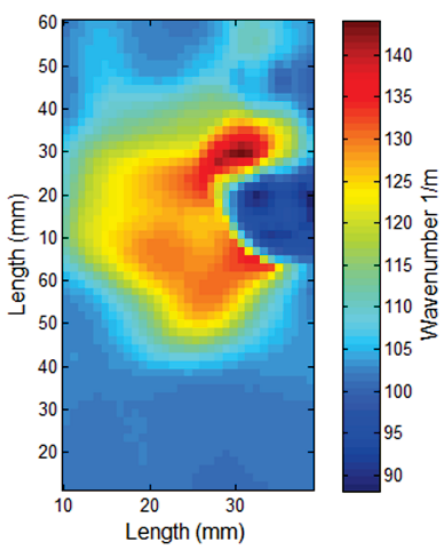

(b)

Figure 10: (a): Local wavenumber result for the experimental data using a $5 \mathrm{~mm}$ spatial window: The small window size results in higher resolution for capturing spatial changes in wavenumber, but it cannot accurately capture the wavenumber since the window is smaller than the A0 wavelength and (b): Local wavenumber result for the experimental data using a $20 \mathrm{~mm}$ spatial window: The larger window size results in a more accurate measure of wavenumber, but has low resolution for spatial changes in wavenumber. 


\section{CONCLUSIONS}

In this paper, two tools measuring spatially varying wavenumbers in guided wavefield data have been defined and applied to both numerical and experimental data sets. In the simplest cases, those metrics can be directly converted into Effective Thickness, which is a representation of the depth of subsurface features created using dispersion relations. As shown in the results, both techniques perform well at providing adequate resolution spatial wavenumber maps when the guided wave mode wavelength is smaller than the damage size. When the guided wave mode wavelength is on the order of the damage size, the IW technique appears to out-perform the LW approach. The LW technique is also more computationally intensive than the IW approach since it requires 3D FFTs. Both approaches require somewhat subjective choices to be made during their implementation. For the LW technique a time gate and a spatial window size (which has a significant effect on the resulting wavenumber map) must be chosen. For the frequency domain IW technique a time gate must be chosen (in the time domain IW technique a specific snapshot in time must be chosen). The two methodologies are a first step toward a hybrid SHM/NDE approach for damage assessment in composites. Based on these initial results, both techniques appear promising for providing damage characterization information, particularly for delamination damage in composite laminates. In future work we plan to investigate the advantages and limitations of these techniques for damage characterization.

\section{REFERENCES}

[1] H. Sohn, D. Dutta, J. Yang, H. Park, M. DeSimio, and E. Swenson, "Delamination detection in composites through guided wave field processing," Composites Science and Technology 71, pp. 1250-1256, 2011.

[2] D. Barnoncel, W. J. Staszewski, J. Schell, and P. Peres, "Damage detection in reusable launch vehicle components using guided ultrasonic waves and 3d laser vibrometry," Proc. SPIE 8695, p. 86950D, 2013.

[3] M. Rogge and R. Parker, "Instantaneous wavenumber estimation of guided wavefield images for characterization of damage in composite structures," NASA TM, 2013.

[4] M. Rogge and C. A. Leckey, "Characterization of impact damage in composite laminates using guided wavefield imaging and local wavenumber domain analysis," Ultrasonics 53, pp. 1217-1226, 2013.

[5] C. Leckey, M. Rogge, and R. Parker, "Guided waves in anisotropic and quasi-isotropic aerospace composites: Three-dimensional simulation and experiment," Ultrasonics 54, pp. 385-394, 2014.

[6] M. Carrara and M. Ruzzene, Numerical and experimental analysis of guided waves propagation in composite plates, 2013. 